\title{
Block Circulant Decomposition of Cross-Correlation Matrix for Transmit MIMO Beamforming
}

\author{
Christian Hammes ${ }^{* \dagger}$, Bhavani Shankar M. R.* and Björn Ottersten* ${ }^{*}$ University of Luxembourg, SnT, ${ }^{\dagger}$ Email: \\ christian.hammes@uni.lu
}

\begin{abstract}
This paper comprises the transmit probing signal design under the trade-off between good target discrimination (low cross-correlation beam pattern) and beam pattern design (desired auto-correlation beam pattern) in a Multiple-InputMultiple-Output (MIMO) configuration. The quartic optimization problem, with an finite alphabet constraint on the probing signal by using Quadrature Phase Shift Keying (QPSK) in a multiplexed antenna system, is solved by a Fourier series approximation of the desired beam pattern by exploiting a block circulant property of the transmit signal matrix. The analytical evaluation of the mean square error between an ideal and the proposed cross-correlation beam pattern is $-35 \mathrm{~dB}$.
\end{abstract}

\section{INTRODUCTION}

The design of a probing signal in a Multiple-Input-MultipleOutput (MIMO) configuration is still an open topic and extensively discussed in literature [1]-[14]. A special challenge is the design of a desired auto-correlation beam pattern, while keeping good cross-correlation beam pattern properties [10]. The beam pattern investigation is based on the covariance matrix of the transmitted probing signals. Therefore, the authors in [1]-[7], [10] consider a two stage design, where the first stage is the optimization of the covariance matrix and the second stage is the determination of the probing signals, which satisfy the covariance structure of stage one. Only in [11], the probing signal is determined by a one stage optimization.

The approaches in literature with regards to find an optimal solution for a given desired radiation pattern are very diverse, but all of the authors [1]-[7] have the objective of designing probing signals under certain constrains with low computational complexity. The works [3] and [6] are motivated by the design of Finite Impulse Response (FIR) filters and transfer parameters like transition band and or ripple height towards the beam pattern design. The authors in [2] relax the optimization problem in an least square problem, which is easier to solve than the original one. Another approach of enhancing the computational complexity is the analytical evaluation of an integral like objective function [1]. In [7] the correlation matrix is decomposed in a spatial and temporal division matrix, while maintaining good auto- and cross-correlation properties of the probing signal. Very often, the objective function is quartic with regards to the probing signal. A cyclic algorithm for solving such quartic problems under constant modulus and low peak to average ratio constraint is proposed in [4].

A constant modulus constraint is found quite often in literature [3], [4] and [7]. Solving a quartic optimization problem under constant modulus constraint is treated in [8]- [12]. The diversity of different algorithm shows the complexity of such kind optimization problems.
The authors in [15]-[18] investigate the beam pattern optimization problem more from an antenna group factor perspective, rather than a signal point of view. The major difference lies in the investigation of radio frequency switches or multiplexers leading to on/off modulation, which is different from constant modulus sequences. Further, discrete phase modulation is also included in [15], leading to difficult optimization problem which is solved with a differential evolution algorithm.

It can be summarized, that the computational complexity is one of the biggest issues in adaptive beam pattern design. The computational complexity is mainly justified in quartic optimization problems with subject to a finite alphabet constraint. With regards to a MIMO configuration, low cross-correlation beam pattern is crucial when a good target discrimination is desired [10]. The contributions of this work are the following:

- linear system model for inter pulse modulation

- deterministic and therefore real time capable algorithm with no iterations by exploiting the Fourier transform like property of ULA's

- low cross-correlation beam pattern characteristic by proposing a block circulant probing signal matrix

- a solution for an optimization problem under the constraint of two Quadrature Phase Shift Keying (QPSK) channels in transmit ULA configuration with arbitrary amount of antennas

In this work the operator $\|\cdot\|_{p}$ defines the $l_{p}$-norm. A matrix entry is defined by $[\cdot]_{\eta, \gamma}$, where $\eta$ denotes the row index and $\gamma$ the column index. A vector entry $[\cdot]_{\eta}$ is defined by one index $\eta$. The $E\{\cdot\}$ denotes the expectation operator. The set of complex numbers is defined as $\mathbb{C}$, while $j=\sqrt{-1}$ represents the complex number. The Kronecker product is defined as $\otimes$.

\section{SySTEM MODEL}

The underlying system comprises one local oscillator, generating a train of $\mathcal{I}_{c}$ Frequency Modulated Continuous Wave (FMCW) pulses. Each pulse has a duration of $T_{c}$, bandwidth $B$ with the center frequency $f_{0}=\frac{\omega_{0}}{2 \pi}$. This train of FMCW pulses is further processed by the transmit modulation unit. This unit comprises $N_{c}$ parallel QPSK modulators, which are further multiplexed between the $N_{T}$ transmit antennas, where the number of channels is less than the number of transmit antennas, leading to $N_{c}<N_{T}$.

The sparse transmit Uniform Linear Array (ULA), with an antenna inter-element spacing of $d_{T}$, transmits the modulated signals towards $K$ distinct targets. The back scattered signal from $K$ targets are superposing in space and are captured by the $N_{R}$ receive antenna. The $N_{R}$ receive antennas are 
further mounted as a ULA with an inter-element spacing of $d_{R}=\frac{\lambda}{2}$. This configuration leads to a Multiple- InputMultiple-Output (MIMO) antenna configuration, with a sparse transmit $d_{T}=N_{R} d_{R}$ and dense receive ULA, leading to an investigation of virtual MIMO array.

After receiving, the $N_{R}$ different receive signals are mixed down and converted to the digital domain using Analog to Digital Converter (ADC), where the receive signal processing is done by matched filtering.

\section{A. Transmitted Signal}

Since the receiver has access to sampled waveforms, a discrete time representation of the transmitted signal is considered. Each FMCW pulse $\mathbf{p} \in \mathbb{C}^{\mathcal{I}_{s} \times 1}$ is therefore sampled with the sampling time $T_{s}$ with a of $\mathcal{I}_{s}$ samples [23]. Another reasonable assumption is that the phase modulation units as well as the multiplexing are operating much slower than the sampling time $T_{s}$, such that the transmit modulation is done across pulses, leading to an inter pulse modulation. For each FMCW pulse, $N_{T}$ transmit antennas need to be modulated leading to the signal modulation vector $\mathbf{s} \in \mathbb{C}^{\mathcal{I}_{c} N_{T} \times 1}$. The multiplexing restricts the signal modulation vector $\mathrm{s}$ to $N_{c} \mathcal{I}_{c}$ nonzero entries. In addition to the zero entries, $\mathbf{s}$ is restricted to a QPSK modulation, leading to $\mathbf{s} \in \Omega_{0}^{\mathcal{I}_{c} N_{T} \times 1}$, where the set $\Omega_{0}=\{0\} \cup \Omega$ comprises the zero to model the multiplexing and $\Omega=\{1,-1, j,-j\}$ represents the fourth root of the complex unit circle due to the QPSK modulation. The transmit signal vector $\mathbf{x} \in \mathbb{C}^{\mathcal{I}_{s} \mathcal{I}_{c} N_{T} \times 1}$ comprises the signal modulation vector and the pulse vector,

$$
\mathbf{x}=\mathbf{s} \otimes \mathbf{p}
$$

\section{B. System Transfer Function and Received Signal}

Due to the inter pulse modulation and assuming the propagation delay is much smaller than the pulse duration $T_{c}$, the propagation delay just influences the pulse vector. Further, the pulse vector is completely separable from the signal modulation vector, as induced by the Kronecker product in (1). The down mixed and digitized pulse vector for the $\kappa$-th target is defined as $\hat{\mathbf{p}}_{\kappa} \in \mathbb{C}^{\mathcal{I}_{s} \times 1}$. The overall received signal can be written as,

$$
\hat{\mathbf{y}}=\sum_{\kappa=1}^{K}\left(\mathbf{H}_{\kappa} \mathbf{s}\right) \otimes \hat{\mathbf{p}}_{\kappa} .
$$

The matrix $\mathbf{H}_{\kappa}$ includes the MIMO channel characteristic for the $\kappa$-th target, the diagonal Doppler shift matrix $\mathbf{D}_{\omega_{D \kappa}}$ and the attenuation factor $\alpha_{\kappa}$, comprising target Radar Cross Section (RCS), path loss and the attenuation due to target range. Therefore, $\kappa$-th target channel matrix can be written as,

$$
\mathbf{H}_{\kappa}=\alpha_{\kappa}\left(\mathbf{a}_{R \kappa} \mathbf{a}_{T \kappa}^{H}\right) \otimes \mathbf{D}_{\omega_{D \kappa}} .
$$

Where the receive steering vector with the antenna index $m$ is defined as $\left[\mathbf{a}_{R \kappa}\right]_{m}=\exp \left(j k_{0} \sin \left(\phi_{\kappa}\right) d_{R} m\right)$ The free space wavenumber is defines as $k_{0}=\frac{2 \pi}{\lambda}$ and $\phi_{\kappa}$ defines the $\kappa$-th target angle of arrival. In accordance to the ULA assumption, the transmit steering vector with the antenna index $n$ is defined as $\left[\mathbf{a}_{T \kappa}\right]_{n}=\exp \left(j k_{0} \sin \left(\phi_{\kappa}\right) d_{T} n\right)$. The Doppler information is mathematically separable in the system model, therefore, the Doppler matrix can be modeled as diagonal,

$$
\mathbf{D}_{\omega_{D \kappa}}=\operatorname{diag}\left(\exp \left(j \omega_{D \kappa} T_{c} 1\right), \cdots, \exp \left(j \omega_{D \kappa} T_{c} \mathcal{I}_{c}\right)\right) .
$$

Since the range information can be completely separated from the angle-Doppler information and further this work focuses on beam pattern design, it is sufficient to consider just the system model with the signal modulation vector,

$$
\mathbf{y}=\sum_{\kappa=1}^{K} \mathbf{H}_{\kappa} \mathbf{s}
$$

\section{Matched Filter}

The receive signal processing comprises a matched filter approach whose coefficients take the form $\mathbf{y}_{M}=\mathbf{H}_{M} \mathbf{s}$. The matched filter coefficients do not include any RCS information and are parametrized by angle $\phi_{M}$ and Doppler shift $\omega_{D M}$,

$$
\mathbf{H}_{M}=\left(\mathbf{a}_{R M} \mathbf{a}_{T M}^{H}\right) \otimes \mathbf{D}_{\omega_{D M}} .
$$

Due to the inter pulse modulation scheme, it is a reasonable assumption to model the $\kappa$-th attenuation factor $\alpha_{\kappa}$ as a Swerling one model, where each target RCS fluctuates across pulses and have to be considered as statistical parameters [22]. In order to get an estimate of the matched filter output, the expected value of the squared matched filter output is investigated,

$$
E\left\{\left|\mathbf{y}_{M}^{H} \mathbf{y}\right|^{2}\right\}=E\left\{\left|\sum_{\kappa=1}^{K} \mathbf{s}^{H} \mathbf{H}_{M}^{H} \mathbf{H}_{\kappa} \mathbf{s}\right|^{2}\right\} .
$$

The block diagonal structure of $\mathbf{H}_{M}$ yields to a sum over $\mathcal{I}_{c}$ pulses, where the pulse index is denoted by $i_{c}$. Further, it can be assumed that RCS fluctuations, due to the Swerling one model, are statistically independent from each other, leading to the following matched filter output expression,

$$
E\left\{\left|\mathbf{y}_{M}^{H} \mathbf{y}\right|^{2}\right\}=\sum_{\kappa=1}^{K} \sigma_{\kappa}^{2}\left|\mathbf{a}_{R M}^{H} \mathbf{a}_{R \kappa}\right|^{2}\left|P\left(\phi_{\kappa}, \phi_{M}\right)\right|^{2} .
$$

The attenuation factor variance for the $\kappa$-th target is defined as $\sigma_{\kappa}$. In the following investigations, Doppler influence is neglected, because its investigation exceeds the scope of this paper. Under the zero Doppler assumption, the following equation describes cross-correlation beam pattern,

$$
P\left(\phi_{\kappa}, \phi_{M}\right)=\mathbf{a}_{T M}^{H}\left(\sum_{i_{c}=1}^{\mathcal{I}_{c}} \tilde{\mathbf{s}}_{i_{c}} \tilde{\mathbf{s}}_{i_{c}}^{H}\right) \mathbf{a}_{T \kappa}
$$

The transmit antenna modulation vector for the $i_{c}$-th pulse is denoted as $\tilde{\mathbf{s}}_{i_{c}} \in \Omega_{0}^{N_{T} \times 1}$. For $\phi_{\kappa}=\phi_{M}$, (9) represents the transmitted auto-correlation beam pattern. If the covariance matrix of the transmitted signal $\mathbf{R}_{\tilde{s}} \in \mathbb{C}^{N_{T} \times N_{T}}$ is defined as,

$$
\mathbf{R}_{\tilde{s}}=\sum_{i_{c}=1}^{\mathcal{I}_{c}} \tilde{\mathbf{s}}_{i_{c}} \tilde{\mathbf{s}}_{i_{c}}^{H}=\tilde{\mathbf{S}} \tilde{\mathbf{S}}^{H}
$$

the probing signal matrix is defined as $\tilde{\mathbf{S}}=$ $\left(\begin{array}{lllll}\tilde{\mathbf{s}}_{1} & \cdots & \tilde{\mathbf{s}}_{i_{c}} & \cdots & \tilde{\mathbf{s}}_{\mathcal{I}_{c}}\end{array}\right) \in \mathbb{C}^{N_{T} \times \mathcal{I}_{c}}$. Based on the derivation of this section, the an optimization criteria can be defined in the following. 


\section{BeAm Pattern Optimization AND AdAPtation}

As derived in Section II, the matched filter output takes the form of a two dimensional radiation pattern, where the actual target position $\phi_{\kappa}$ is mapped to the matched filter output $\phi_{M}$. The matched filter operates optimal, if the actual target position appears at the matched filter output as a Dirac impulse, leading to the desired radiation pattern $P_{d}\left(\phi_{\kappa}, \phi_{M}\right)$,

$$
P_{d}\left(\phi_{\kappa}, \phi_{M}\right)=\left\{\begin{array}{ll}
P_{d}(\phi) & \text { for } \phi_{\kappa}=\phi_{M} \\
0 & \text { else }
\end{array} .\right.
$$

The formulation above means, the auto-correlation beam pattern is desired to have an arbitrary form of $P_{d}(\phi)$, while the cross-correlation beam pattern is supposed to be zero, namely the matched filter output is not disturbed by any clutter. If the desired radiation pattern $P_{d}\left(\phi_{\kappa}, \phi_{M}\right)$ is uniformly sampled in both dimensions $\phi_{\kappa}$ and $\phi_{M}$ with a total sample number of $N_{\kappa}$ for the $\phi_{\kappa}$ dimension and $N_{M}$ for the $\phi_{M}$ dimension the desired radiation pattern can be written in matrix notation $\mathbf{P}_{d} \in \mathbb{R}^{N_{M} \times N_{\kappa}}$. Further, since the desired cross-correlation beam pattern is sampled, the transmit steering vector within the matched filter $\mathbf{a}_{T M}$ and the target transmit steering vector $\mathbf{a}_{T \kappa}$ have to be sampled as well leading to matched filter transmit steering matrix $\mathbf{A}_{T M}=\left(\mathbf{a}_{T M_{1}} \cdots \mathbf{a}_{T M_{N_{M}}}\right)^{T} \in$ $\mathbb{C}^{N_{M} \times N_{T}}$ and a target transmit steering matrix $\mathbf{A}_{T \kappa}=$ $\left(\mathbf{a}_{T \kappa_{1}} \cdots \mathbf{a}_{T \kappa_{N_{\kappa}}}\right)^{T} \in \mathbb{C}^{N_{\kappa} \times N_{T}}$. Therefore, the signal design problem can be formulated as follows,

$$
\begin{gathered}
\min _{\tilde{\mathbf{S}} \in \Omega_{0}^{N_{T} \times \mathcal{I}_{c}}}\left\|\mathbf{P}_{d}-\mathbf{A}_{T M}^{H} \mathbf{R}_{\tilde{s}} \mathbf{A}_{T \kappa}\right\|_{\mathcal{F}}^{2} . \\
\text { s. t. }\left\|\tilde{\mathbf{s}}_{i_{c}}\right\|_{0}=N_{c}
\end{gathered}
$$

The zero norm, as a constraint, represents the multiplexing of $N_{c}=2$ towards $N_{T}$ antennas. Further, the probing signal matrix $\tilde{\mathbf{S}}$ is constraint to a finite alphabet $\Omega_{0}$. The optimization problem is in general hard to solve. In order to overcome the difficulties in the solution, the ULA assumption is exploited towards providing a simple, albeit sub-optimal, framework for designing $\tilde{\mathbf{S}}$ to enhance the cross-correlation beam pattern.

\section{A. Block Circulant Property and Target Discrimination}

The assumption of a transmit ULA yields a Vandermonde matrix for $\mathbf{A}_{T M}$ and $\mathbf{A}_{T \kappa}$. If the covariance matrix $\mathbf{R}_{s}$ has Toeplitz structure, a Vandermonde decomposition can be applied, leading to a Vandermonde-Diagonal-Vandermonde matrix structure [20]. Further, if the covariance matrix is circulant Hermitian matrix, the aforementioned decomposition is, in fact, the eigenvalue decomposition with the columns of Discrete Fourier Transform (DFT) matrix being the eigenvectors of $R_{\tilde{s}}$ [21]. A circulant Hermitian matrix can be constructed by using a block circulant probing signal matrix,

$$
\begin{aligned}
\tilde{\mathbf{S}} & =\left(\begin{array}{lllll}
\tilde{\mathbf{s}}_{1} & \cdots & \tilde{\mathbf{s}}_{i_{c}} & \cdots & \tilde{\mathbf{s}}_{\mathcal{I}_{c}}
\end{array}\right) \\
& =\left(\begin{array}{lllll}
\mathbf{B}_{1} & \cdots & \mathbf{B}_{b} & \cdots & \mathbf{B}_{N_{B}}
\end{array}\right) .
\end{aligned}
$$

Each block circulant matrix $\mathbf{B}_{b} \in \mathbb{C}^{N_{T} \times N_{T}}$ can be parametrized by a single column vector $\mathbf{c}_{b} \in \mathbb{C}^{N_{T} \times 1}$ [21]. The advantage of restricting the covariance matrix to be circulant instead of just Toeplitz is that the eigenvector matrix
$\mathbf{D}=\left(\mathbf{d}_{1} \cdots \mathbf{d}_{n} \cdots \mathbf{d}_{N_{T}}\right) \in \mathbb{C}^{N_{T} \times N_{T}}$ is a DFT matrix with the eigenvectors $\mathbf{d}_{n}$, which is orthonormal,

$$
\mathbf{R}_{\tilde{s}}=\sum_{b=1}^{N_{B}} \mathbf{B}_{b} \mathbf{B}_{b}^{H}=\mathbf{D}\left(\sum_{b=1}^{N_{B}} \boldsymbol{\Psi}_{b} \boldsymbol{\Psi}_{b}^{H}\right) \mathbf{D}^{H}=\mathbf{D} \boldsymbol{\Lambda} \mathbf{D}^{H} .
$$

The orthonormal property of the DFT matrix yields a covariance matrix with a DFT eigenvector matrix. The diagonal eigenvalue matrix of the covariance matrix $\boldsymbol{\Lambda} \in \mathbb{R}^{N_{T} \times N_{T}}$ is a squared sum over all eigenvalue matrices $\Psi_{b} \in \mathbb{C}^{N_{T} \times N_{T}}$ of the block circulant probing signal matrix.

The general radiation pattern for any block circulant probing signal matrix leads to the following,

$$
\begin{aligned}
P\left(\phi_{\kappa}, \phi_{M}\right) & =\mathbf{a}_{T M}^{H} \mathbf{D} \boldsymbol{\Lambda} \mathbf{D}^{H} \mathbf{a}_{T \kappa} \\
& =\sum_{n=1}^{N_{T}} \lambda_{n} f\left(k_{\phi_{\kappa}}-n \frac{2 \pi}{N_{T}}\right) f\left(k_{\phi_{M}}-n \frac{2 \pi}{N_{T}}\right) .
\end{aligned}
$$

Where the discrete sinus cardinal function is defined as $f(x)=\operatorname{sinc}_{d}\left(\frac{N_{T}}{2} x\right)=\frac{\left.\sin \left(\frac{N_{T}}{2} x\right)\right)}{\sin \left(\frac{1}{2} x\right)}$, the target and matched filter wavenumber are defines as $k_{\phi_{\kappa}}=k_{0} d_{T} \sin \left(\phi_{\kappa}\right)$ and $k_{\phi_{M}}=k_{0} d_{T} \sin \left(\phi_{M}\right)$, respectively, and $\lambda_{n}$ represents the $n$ th eigenvalue of $\mathbf{R}_{\tilde{s}}$. It is stated in [19], that the maximum resolution for equal antenna element power constraint in an virtual MIMO configuration is achieved, when the transmitted signals are perfectly orthogonal, leading to diagonal covariance matrix,

$$
\begin{aligned}
& P_{\text {ort }}\left(\phi_{\kappa}, \phi_{M}\right)=\mathbf{a}_{T M}^{H} \mathbf{R}_{\tilde{s}} \mathbf{a}_{T \kappa} \\
& =\operatorname{tr}\left(\mathbf{R}_{\tilde{s}}\right) \operatorname{sinc}_{d}\left(\frac{N_{T}}{2} k_{0} d_{T}\left(\sin \left(\phi_{M}\right)-\sin \left(\phi_{\kappa}\right)\right)\right) .
\end{aligned}
$$

Since in (16) the physical resolution limit of the presented structure is shown, it is of interest how the proposed approach in (15) performs in relation to (16). This result is further discussed through simulations.

\section{B. Probing Signal Generation and Beam Pattern Design}

As a result of the previous section, the cross-correlation beam pattern is addressed by applying a block circulant property to the probing signal matrix and its eigenvector structure. The auto-correlation property on the other hand lies within the eigenvalues, which can be proved by setting $\phi_{M}=\phi_{\kappa}$ in (15),

$$
P(\phi)=\sum_{n=1}^{N_{T}} \mathbf{v}_{n}^{H} \mathbf{R}_{c} \mathbf{v}_{n} f\left(k_{\phi}-n \frac{2 \pi}{N_{T}}\right)^{2} .
$$

The auto-correlation beam pattern $P(\phi)$ is therefore a convolution of $\mathbf{v}_{n}^{H} \mathbf{R}_{c} \mathbf{v}_{n}$ and $f\left(k_{\phi}-n \frac{2 \pi}{N_{T}}\right)^{2}$, where the latter functions is given due to the block circulant construction. The first term $\mathbf{v}_{n}^{H} \mathbf{R}_{c} \mathbf{v}_{n}$ comprises the auto correlation matrix $\mathbf{R}_{c}=$ $\sum_{b=1}^{N_{B}} \mathbf{c}_{b} \mathbf{c}_{b}^{H}$ and the Fourier vector $\left[\mathbf{v}_{n}\right]_{q}=\exp \left(j \frac{2 \pi}{N_{T}} q n\right)$, which further leads to,

$$
\mathbf{v}_{n}^{H} \mathbf{R}_{c} \mathbf{v}_{n}=\sum_{b=1}^{N_{B}} 2+2 \cos \left(\frac{2 \pi}{N_{T}} \tau_{b} n+\varphi_{b}\right) .
$$

The frequency $\frac{2 \pi}{N_{T}} \tau_{b}$ of the cosine function in (18) is determined by the relative position $\tau_{b}$ of the two non zero elements 

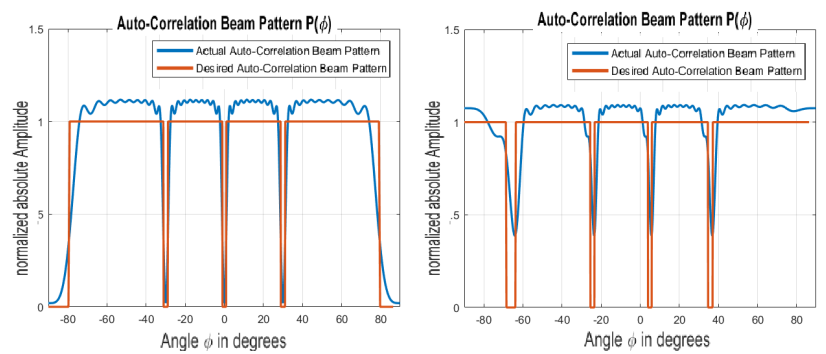

Fig. 1: Auto-Correlation Beam Pattern for a desired Target suppression at $\phi=5^{\circ}$. The periodicity is related to the sparsity of the transmit array $d_{T}=N_{R} \frac{\lambda}{2}$, while the offset is related to superposition of squared sine and cosine functions.

in $\mathbf{c}_{b}$, therefore $\tau_{b}$ is limited to $N_{T}-1$ due to the size of $\mathbf{c}_{b}$. The phase shift $\varphi_{b}$ denotes the relative phase shift between the two non zero elements in $\mathbf{c}_{b}$ leading to a minimum relative phase shift of $\frac{\pi}{2}$ due to the QPSK modulation. Due to the assumption $N_{B} \gg N_{T}$ and due to limited discrete $\tau_{b}$, the sum in (18) can be enrolled and reordered to,

$$
\mathbf{v}_{n}^{H} \mathbf{R}_{c} \mathbf{v}_{n}=2 N_{B}+\sum_{q=-\left(N_{T}-1\right)}^{N_{T}-1} A_{q} \exp \left(j \frac{2 \pi}{N_{T}} q n\right) .
$$

The amplitudes $A_{q} \in \mathbb{C}^{1 \times 1}$ are therefore discrete quantities, satisfying $\sum_{q=1}^{N_{T}-1}\left|\Re\left\{A_{q}\right\}\right|+\left|\Im\left\{A_{q}\right\}\right|=N_{B}$. The phase information is implicitly inside the amplitudes. Further, the condition $A_{q}=A_{-q}^{*}$ has to be satisfied, since $\mathbf{v}_{n}^{H} \mathbf{R}_{c} \mathbf{v}_{n}$ has to be real. At this point, all parameters are known to solve (17) with the help of a inverse Fourier transform and an appropriate algorithm to map all $A_{q}$ to the set of vectors $\mathbf{c}_{b}$. Simulation results proof the feasibility of this approach.

\section{Simulation}

The simulation is carried out with $N_{T}=10$ transmit antennas and $N_{R}=4$ receive antennas. Figure reffig:auto5deg illustrates the feasibility of beam pattern shaping using the proposed approach. The difference between Figures in 1 is that the beam pattern on the right goes not to zero, because the radiation pattern is composed of squared sine and cosine, due to the two channel QPSK modulation scheme. In order to ensure any arbitrary beamshape, like a null steering at $5^{\circ}$ as illustrated in the Figure 1 on the right, a mixture of squared sine and cosine functions is necessary, because an arbitrary angle can be only achieved if both functions are present. The cross-correlation beam pattern 2 on the right illustrates the resolution capability of perfectly orthogonal signals. The cross-correlation beam pattern in Figure 2 left depicts the resolution characteristic for the proposed approach. The resolution characteristic is defined by the width of the diagonal line in Figure 2 right and 2. It can be seen, that the resolution is similar to Figure 2 right with some differences in resolution on the transition band (when the auto-correlation beam pattern goes from low to high level). A cutting plane of Figure 2 at $5^{\circ}$ (similar to a matched filter output for a single target at $5^{\circ}$ ) is illustrated in Figure 3. It can be seen, that the resolution of the proposed approach is a bit curse and

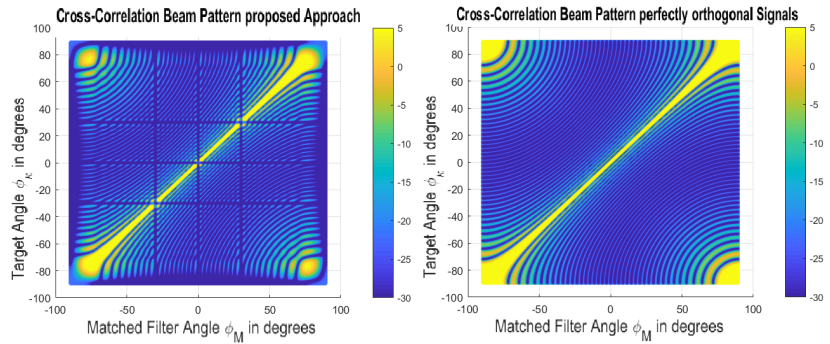

Fig. 2: Cross-Correlation Beam Pattern for the proposed approach $P\left(\phi_{\kappa}, \phi_{M}\right)$ (see (15)), which confirms the similarity to orthogonal signals. The target resolution degrades a bit near the beam pattern transitions.

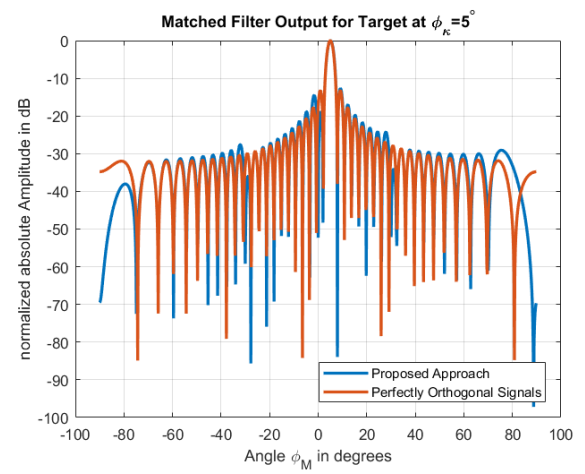

Fig. 3: Matched Filter Output of the proposed approach and perfectly orthogonal signal. The mean square error between both outputs is $M S E=-35 \mathrm{~dB}$.

the sidelobes are a bit higher than the matched filter output for the perfectly orthogonal signals. Nevertheless, the mean square error between the proposed approach and perfectly orthogonal signals is about $M S E=-35 \mathrm{~dB}$, which confirms the good resolution capability of the proposed approach.

\section{CONClusion}

The proposed approach is powerful in terms of the deterministic computational complexity, since no iterative algorithm is needed and therefore there are no convergence issues even with hard constraints on the transmitted signal, like QPSK modulation in an multiplexed antenna structure. The capability of shaping the beam pattern with correlated signals, while keeping the resolution characteristic (cross-correlation beam pattern), makes the proposed method applicable to a virtual MIMO configuration. Further, the proposed approach is applicable to any desired auto-correlation beam pattern, while keeping good resolution properties.

\section{REFERENCES}

[1] T. Aittomaki and V. Koivunen Low-Complexity Method For Transmit Beamforming in MIMO Radars, IEEE Proceedings in International Conference on Acoustics, Speech and Signal Processing (ICASSP) 2007.

[2] T. Aittomaki and V. Koivunen Signal Covariance Matrix Optimization for Transmit Beamforming in MIMO Radars, Asilomar Conference on Signals, Systems and Computers 2007.

[3] D. Fuhrmann and G. San Antonio Transmit Beamforming for MIMO Radar Systems using Signal Cross-Correlation, IEEE Trans. on Aerospace and Electronic Systems vol. 44, no. 1 January 2008. 
[4] P. Stoica, J. Li and X. Zhu Waveform Synthesis for Diversity-Based Transmit Beampatter Design, IEEE Trans. Signal Process. vol. 56, no. 6 June, 2008.

[5] S. Ahmed, J, Thompson, Y. Petillot and B. Mulgrew Unconstraint Synthesis of Covariance Matrix for MIMO Radar Transmit Beampattern, IEEE Trans. Signal Process. vol. 59, no. 8 August, 2011.

[6] G. Hua and S. Abeysekera MIMO Radar Transmit Beampattern Design With Ripple and Transition Band Control, IEEE Trans. Signal Process. vol. 61, no. 11 June, 2013.

[7] T. Yang, T. Su, W. Zhu and H. Wu Transmit waveform synthesis for MIMO radar using spatial-temporal decomposition of correlation matrix, IEEE Proceedings in Radar Conference 2014.

[8] P. Stoica, H. He and J. Li New Algorithms for Designing Unimodular Sequences With Good Correlation Properties, IEEE Trans. Signal Process. vol. 57, no. 4 April, 2009.

[9] M. Soltanalian and P. Stoica Computational Design of Sequences With Good Correlation Properties, IEEE Trans. Signal Process. vol. 60, no. 5 May 2012.

[10] P. Stoica, J.Li and Y. Xie On Probing Signal Design For MIMO Radar, IEEE Trans. Signal Process. vol. 55, no. 8 August 2007.

[11] M. Soltanalian, H. Hu and P. Stoica Single-stage transmit beamforming design for MIMO radar, Elsevier Signal Processing 102 132-138 2014

[12] M. Soltanalian and P. Stoica Designing Unimodular Codes Via Quadratic Optimization, IEEE Trans. Signal Process. vol. 62, no. 5 March 2014.

[13] M. Soltanalian, B. Tang, J. Li and P. Stoica Joint Design of the Receive Filter and Transmit Sequence for Active Sensing, IEEE Signal Processing Letters vol. 20, no. 5 May 2013.

[14] J.Li, P. Stoica and X. Zheng Signal Synthesis and Receiver Design for MIMO Radar Imaging, IEEE Trans. Signal Process. vol. 56, no. 8 August 2008.

[15] J. Yang, W. Li and X. Shi Phase Modulation Tequnique for FourDimensional Arrays, IEEE Antennas and Wireless Propagation Letters vol. 132014

[16] P. Rocca, L. Poli, G. Oliveri and A. Massa Adaptive Nulling in Time-Varying Scenarios Through Time-Modulated Linear Arrays, IEEE Antennas and Wireless Propagation Letters vol. 112012.

[17] Y. Tong and A. Tennant A Two-Channel Time Modulated Linear Array With Adaptive Beamforming, IEEE Transactions on Antennas and Propagation vol. 60, no. 1 January 2012.

[18] J. Guo, S. Qu, J. Hu and Z. Nie A Study on Linear Frequency Modulation Signal Transmission by 4-D Antenna Arrays, IEEE Transactions on Antennas and Propagation vol. 63, no. 12 December 2015.

[19] D. Bliss, K. Forsythe, G. Fawcett, "MIMO Radar: Resolution, Performance, and Waveforms," in Proceedings of ASAP, 2006.

[20] Z. Yang, L. Xie and P. Stoica Vandermonde Decomposition of Multilevel Toeplitz Marices With Application to Multidimensional Super-Resolution, IEEE Transactions on Information Theory 2016.

[21] R. Gray Toeplitz and Circulant Matrices: A review, Deptartment of Electrical Engineering Stanford University

[22] M. B. Kilani, Y. Nijsure, G. Gagnon, G. Kaddoum, F.Gagnon, Cognitive waveform and receiver selection mechanism for multistatic radar, IET Radar, Sonar and Navigation, 2015.

[23] C. Hammes, B. Shankar, Y. Nijsure, T. Spielmann and B. Ottersten, Random Phase Center Motion Technique for Enhanced Angle-Doppler Discrimination Using MIMO Radars, IEEE European Signal Processing Conference, 2017. 